\title{
Optimizing small molecules as a chemical tool to control DNA Repair enzymes using X-ray crystallography and computational techniques
}

\author{
Davide Moiani ${ }^{1}$ and John A. Tainer ${ }^{1,2}$. \\ 1 The University of Texas, M.D. Anderson Cancer Center, Houston, TX, United \\ States, Department of Molecular and Cellular Oncology; 2 Molecular Biophysics \\ and Integrated Bioimaging, Lawrence Berkeley National Laboratory, Berkeley, \\ CA, United States.
}

DNA Repair enzymes are involved in several cellular pathways that are initiated by determinate activities. Here, we present a way to use X-ray Crystallography and other computational techniques to arrive at a rational design of small molecules, which act as chemical tools to dissect protein functions and promote the switching among different pathways. Our preliminary studies are transferring best compounds into preclinical study to be used as potential therapy in oncology or neurodegenerative disease. Practical examples in our laboratory are targeting MRE11 and MutSbeta. 